\title{
Electrostatic and electromagnetic instabilities associated with electrostatic shocks: two-dimensional particle-in-cell simulation
}

\author{
Tsunehiko N. Katd® and Hideaki Takabe \\ Institute of Laser Engineering, Osaka University, \\ 2-6 Yamada-oka, Suita, Osaka 565-0871, Japan
}

(Dated: October 9, 2018)

\begin{abstract}
A two-dimensional electromagnetic particle-in-cell simulation with the realistic ion-to-electron mass ratio of 1836 is carried out to investigate the electrostatic collisionless shocks in relatively highspeed $\left(\sim 3000 \mathrm{~km} \mathrm{~s}^{-1}\right)$ plasma flows and also the influence of both electrostatic and electromagnetic instabilities, which can develop around the shocks, on the shock dynamics. It is shown that the electrostatic ion-ion instability can develop in front of the shocks, where the plasma is under counterstreaming condition, with highly oblique wave vectors as was shown previously. The electrostatic potential generated by the electrostatic ion-ion instability propagating obliquely to the shock surface becomes comparable with the shock potential and finally the shock structure is destroyed. It is also shown that in front of the shock the beam-Weibel instability gradually grows as well, consequently suggesting that the magnetic field generated by the beam-Weibel instability becomes important in long-term evolution of the shock and the Weibel-mediated shock forms long after the electrostatic shock vanished. It is also observed that the secondary electrostatic shock forms in the reflected ions in front of the primary electrostatic shock.
\end{abstract}

\section{INTRODUCTION}

Collisionless shock is one of the most interesting phenomena in plasma physics; it dissipates the kinetic energy of the plasma flow into the thermal energy and the electromagnetic energy not by the Coulomb collision but by the collective effect associated with the electric and the magnetic fields. For example, the universe is filled with hot, tenuous collisionless plasmas and a variety of collisionless shocks are produced due to violent phenomena, such as supernova explosions. These shocks are believed to accelerate charged particles to high energies to generate cosmic rays.

The electrostatic shock [1], or the ion-acoustic shock 2], is also one of the collisionless shocks and it forms in unmagnetized collisionless electron-ion plasmas if the Mach number is not so large and the temperature ratio of electrons to ions is relatively large [3]. The electrostatic shocks were observed in various experiments with double-plasma devices [4 [6], with Q-machines [7], with photo-ionized plasmas [8], and with laser plasmas [9, 10]. Recent experiments with intense lasers also showed a possible formation of the electrostatic shock at a high shock speed of $\sim 1000 \mathrm{~km} \mathrm{~s}^{-1}$ [1]. In space, these shocks are observed, for example, in the auroral zone of the Earth [12] as well. In the astrophysical context, there are no clear observations of the electrostatic shocks so far. However, they can also be driven in the universe at a wide range of the plasma flow speed. From the theoretical point of view, the electrostatic shock has been investigated with hybrid or particle-in-cell (PIC) simulations extensively [e.g., 3, 13, 14]. Recent simulations also showed a possible formation of very high Mach number

\footnotetext{
*E-mail: kato-t@ile.osaka-u.ac.jp
}

electrostatic shocks [15].

The requirement of the relatively high electron-to-ion temperature ratio for the electrostatic shocks is also one of the conditions for the electrostatic ion-ion instability [16-19], which is also called the ion/ion acoustic instability, to develop. Since there exist the reflected ions in front of the electrostatic shocks, this instability can grow to generate electrostatic waves there. The wave vector of the instability is oblique or even almost perpendicular to the streaming direction [20, 21] and therefore multidimensional simulations are necessary to investigate the effect of the instability. Although most of the simulations were carried out in one dimension and therefore could not deal with the instability, Karimabadi, Omidi \& Quest [22] carried out a two-dimensional electrostatic hybrid simulation and showed that the electrostatic ionion instability indeed develops in front of the electrostatic shock and affects the structure of the shock significantly. (It should be noted that recently Ohira \& Takahara [23] showed that this instability can also develop in the foot region of the magnetized collisionless shocks in supernova remnants.)

In astrophysical plasmas and laboratory plasmas with recent laser facilities, flows of collisionless plasma whose velocity is even faster than $1000 \mathrm{~km} \mathrm{~s}^{-1}$ can be generated. In such high-speed flows, the electromagnetic instabilities, e.g., the Weibel-type instabilities [24, 25], appears to be important as well as the electrostatic instabilities and can affect the evolution of the electrostatic shocks. The simulation by Karimabadi, Omidi \& Quest 22. was however an electrostatic one and the effects of such electromagnetic instabilities were therefore not included. In addition, since the simulation box size of their simulation was not large compared with the structures generated by the electrostatic instability, larger scale simulations are also desirable.

In this paper, we investigate the electrostatic shocks 
propagating at a relatively high speed with a twodimensional electromagnetic PIC simulation. In particular, we focus on the influence of the electrostatic ion-ion instability and the Weibel-type instabilities on the shock formation.

\section{LINEAR ANALYSIS}

The electrostatic shocks can form in two counterstreaming collisionless plasmas with a relatively high electron-to-ion temperature ratio as shown by the previous simulations [3, 14]. In such situations, the plasma becomes counter-streaming condition in front of the shock and some instabilities (electrostatic and/or electromagnetic) can develop there to affect the shock dynamics. In this section, we present the linear analysis of the electrostatic ion-ion instability and the electromagnetic beam-Weibel instability in counter-streaming plasmas with wave vectors in arbitrary direction.

Consider a counter-streaming plasma consisting of Maxwellian beams; each beam of the species $s$ has the drift velocity of $V_{\mathrm{s}}$ along the $x$-axis and the thermal velocity of $a_{\mathrm{s}}$. The non-perturbed (zeroth order) distribution function for each species is given by

$$
f_{0}^{(\mathrm{s})}\left(v_{x}, v_{y}, v_{z}\right)=\frac{n_{\mathrm{s}}}{\pi^{3 / 2} a_{\mathrm{s}}^{3}} \exp \left[-\frac{\left(v_{x}-V_{\mathrm{s}}\right)^{2}+v_{y}^{2}+v_{z}^{2}}{a_{\mathrm{s}}^{2}}\right],
$$

where $n_{\mathrm{s}}$ is the number density. For this system, the linear dispersion relation of the electrostatic mode is given by

$$
k^{2}+2 \sum_{\mathrm{s}} k_{\mathrm{Ds}}^{2}\left(1+\zeta_{\mathrm{s}} Z\left(\zeta_{\mathrm{s}}\right)\right)=0
$$

where $k$ is the magnitude of the wave vector, $\theta$ is the angle between the wave vector and the $x$-axis,

$$
k_{\mathrm{Ds}} \equiv \omega_{\mathrm{ps}} / a_{\mathrm{s}}, \quad \omega_{\mathrm{ps}} \equiv\left(\frac{4 \pi n_{\mathrm{s}} q_{\mathrm{s}}^{2}}{m_{\mathrm{s}}}\right)^{1 / 2}
$$

and

$$
\zeta_{\mathrm{s}}=\zeta_{\mathrm{s}}(\omega, k, \theta) \equiv\left(\omega / k-V_{\mathrm{s}} \cos \theta\right) / a_{\mathrm{s}}
$$

The function $Z(\zeta)$ called the plasma dispersion function [26] is defined by

$$
Z(\zeta) \equiv \pi^{-1 / 2} \int_{-\infty}^{\infty} \frac{e^{-z^{2}}}{z-\zeta} d z
$$

on the upper half of the complex $\zeta$-plane $(\operatorname{Im}(\zeta)>0)$ and analytically continued into the lower half-plane. Note that the solution of the dispersion relation (2) with $\operatorname{Im}(\omega)>0$ means an unstable mode.

Here, we consider a symmetric Maxwellian beam system consisting of two electron beams $(\mathrm{s}=\mathrm{e}+$ and $\mathrm{e}-)$ and two ion beams ( $\mathrm{s}=\mathrm{i}+$ and $\mathrm{i}-)$ with $n_{e+}=n_{e_{-}}=$ $n_{i+}=n_{i-}=n_{\mathrm{e} 0}, V_{\mathrm{e}+}=V_{\mathrm{i}+}=V$ and $V_{\mathrm{e}-}=V_{\mathrm{i}-}=-V$. Figure 1 shows the linear growth rate of the electrostatic ion-ion instability [16 19], $\Gamma \equiv \operatorname{Im}(\omega)$, numerically calculated for a symmetrical beam system with a relatively high electron-to-ion temperature ratio. For later convenience, we take the following parameters: $m_{\mathrm{i}} / m_{\mathrm{e}}=1836$, $V=0.01 c \sim 3000 \mathrm{~km} \mathrm{~s}^{-1}, T_{\mathrm{e}}=11.3 \mathrm{keV}\left(a_{\mathrm{e}}=0.21 c\right)$, $T_{\mathrm{i}}=1.25 \mathrm{keV}\left(a_{\mathrm{i}}=1.6 \times 10^{-3} c\right)$, and thus $T_{\mathrm{e}} / T_{\mathrm{i}}=9$, where $c$ is the speed of light, $T_{\mathrm{e}}$ and $T_{\mathrm{i}}$ are the electron and the ion temperatures, respectively. The maximum

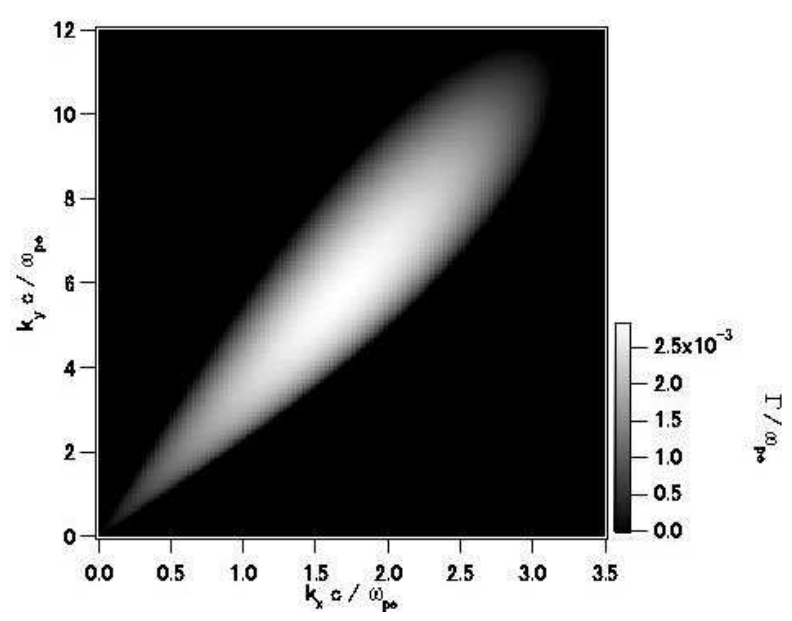

FIG. 1: Linear growth rate of the electrostatic ion-ion instability for the symmetrical beam system of electron-ion plasma with the drift velocity of $0.01 c$ and the electron-to-ion temperature ratio of 9 . The horizontal and the vertical axes are the wave vector in the $x$-direction, $k_{x}$, and that in the $y$-direction, $k_{y}$, respectively. The gray-scale shows the linear growth rate normalized to the electron plasma frequency.

growth rate is obtained as $\Gamma=2.8 \times 10^{-3} \omega_{\text {pe }}$ at $k=$ $6.0 \omega_{\mathrm{pe}} c^{-1}$ and $\theta=74^{\circ}$, where $\omega_{\text {pe }} \equiv\left(4 \pi n_{\mathrm{e} 0} e^{2} / m_{\mathrm{e}}\right)^{1 / 2}$ is the electron plasma frequency defined for the mean electron number density $n_{\mathrm{e} 0}$; therefore the dominant mode is directed almost perpendicular to the streaming direction. The corresponding typical wavelength is given by $\lambda=2 \pi / k=6.7 \times 10^{-1} \lambda_{\mathrm{e}}$, where $\lambda_{\mathrm{e}}=c \omega_{\mathrm{pe}}^{-1}$ is the electron skin depth.

On the other hand, one of the two electromagnetic modes whose electric field lies on the plane made by the $x$-axis and the wave vector can be unstable in this system, too; this is the beam-Weibel instability (or the filamentation instability) [25], which is distinguished from the (ordinary) Weibel instability [24] that grows in a plasma with anisotropic temperatures. The dispersion relation of the beam-Weibel instability is given by

$\omega^{2}-(k c)^{2}+\sum_{\mathrm{s}} \omega_{\mathrm{ps}}^{2}\left[\frac{a_{\mathrm{s}}^{2}+2\left(V_{\mathrm{s}} \sin \theta\right)^{2}}{a_{\mathrm{s}}^{2}}\left(1+\zeta_{\mathrm{s}} Z\left(\zeta_{\mathrm{s}}\right)\right)-1\right]=0$.

Figure 2 shows the linear growth rate of this instability for the same system as in Fig. 1]. The maximum growth rate is obtained as $\Gamma=1.5 \times 10^{-4} \omega_{\mathrm{pe}}$ at $k=0.15 \omega_{\mathrm{pe}} c^{-1}$ (the typical wavelength of $\lambda=4.2 \times 10^{1} \lambda_{\mathrm{e}}$ ) and $\theta=90^{\circ}$. 
Although the growth rate of this beam-Weibel instability is smaller by one order of magnitude than that of the electrostatic ion-ion instability, the magnetic field generated by this instability can be important during the long-term evolution.

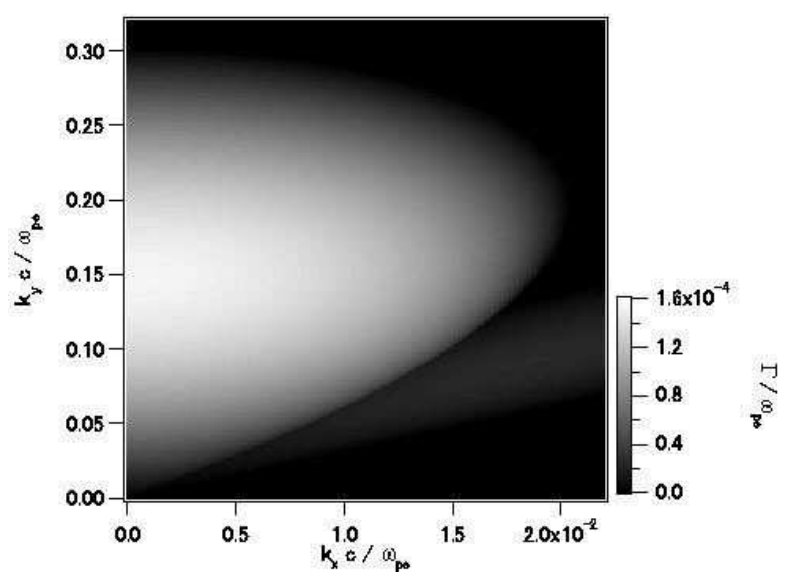

FIG. 2: Linear growth rate of the electromagnetic beamWeibel instability for the same beam system as in Fig. 1.

\section{SIMULATION}

We perform a two-dimensional electromagnetic PIC simulation to investigate the electrostatic shocks together with the instabilities associated with the shock. The simulation code used is a relativistic, electromagnetic, particle-in-cell code with two spatial and three velocity dimensions, namely 2D3V code, developed based on a standard method described in Birdsall \& Langdon [27]; this code was also used to demonstrate the formation of the Weibel-mediated collisionless shocks at relativistic speed in pair plasma 28] and at nonrelativistic speed in electron-ion plasma [29]. Furthermore, it was used to derive the scaling law for the model experiment in laboratory to demonstrate the formation of the Weibelmediated collisionless shocks in laboratory [30] as well. Thus, our code can deal with the electromagnetic modes as well as the (oblique) electrostatic modes. The basic equations of the simulation are the Maxwell's equations and the (relativistic) equation of motion of particles. In the following, the simulation plane is regarded as the $x-y$ plane and the $z$-axis is taken perpendicular to the plane. We take $\omega_{\text {pe }}^{-1}$ as the unit of time and the electron skin depth $\lambda_{\mathrm{e}}=c \omega_{\mathrm{pe}}^{-1}$ as the unit of length (for example, for $n_{\mathrm{e} 0}=1 \mathrm{~cm}^{-3}, \omega_{\mathrm{pe}}^{-1}=1.77 \times 10^{-5} \mathrm{~s}$ and $\lambda_{\mathrm{e}}=5.31 \times 10^{5} \mathrm{~cm}$.) The units of electric and magnetic fields are $E_{*}=B_{*}=c\left(4 \pi n_{\mathrm{e} 0} m_{\mathrm{e}}\right)^{1 / 2}$.

\section{A. Initial condition}

Here, we take the following parameters, which is similar to those of the one-dimensional simulation shown in Fig. 1 (c) of Forslund \& Shonk [14]. The ratio of the ion mass to the electron mass is 1836 . The grid size is $N_{x} \times N_{y}=4096 \times 1024$ and the number of particles is $\sim 54$ per cell per species. The physical size of the simulation box is $L_{x} \times L_{y}=160 \lambda_{\mathrm{e}} \times 40 \lambda_{\mathrm{e}}$ and therefore the size of a cell is $\Delta x=\Delta y \sim 0.039 \lambda_{\mathrm{e}}$. The bulk velocity of the upstream plasma is $V=0.01 c \sim 3000 \mathrm{~km} \mathrm{~s}^{-1}$. The temperatures of the electrons and the ions are $T_{\mathrm{e}}=11.3$ $\mathrm{keV}$ and $T_{\mathrm{i}}=1.25 \mathrm{keV}$, respectively. Thus, the temperature ratio is given by $T_{\mathrm{e}} / T_{\mathrm{i}}=9$. The corresponding thermal velocities are $a_{\mathrm{e}}=0.21 c \sim 6.3 \times 10^{4} \mathrm{~km} \mathrm{~s}^{-1}$ for the electrons and $a_{\mathrm{i}}=1.6 \times 10^{-3} c$ for the ions. The ionacoustic speed is $c_{\mathrm{s}}=\left(k_{\mathrm{B}} T_{\mathrm{e}} / m_{\mathrm{i}}\right)^{1 / 2}=3.5 \times 10^{-3} c$, where $k_{\mathrm{B}}$ is the Boltzmann constant, and the initial bulk Mach number is given by $M_{\text {bulk }} \equiv V / c_{\mathrm{s}}=2.9$. The Debye lengths of the electrons and of the ions are $\lambda_{\mathrm{De}}=0.15 \lambda_{\mathrm{e}}$ and $\lambda_{\mathrm{Di}}=0.05 \lambda_{\mathrm{e}}$, respectively. Initially, the electric and magnetic fields are zero over the simulation box. The boundary conditions for both the particles and the electromagnetic field are periodic in the $y$-direction.

In the simulation, a collisionless shock is driven according to the injection method, or the piston method. There are two walls at the left-hand side (smaller $x$ ) and the right-hand side (larger $x$ ) of the simulation box and these walls reflect particles specularly. Initially, both the electrons and the ions are loaded uniformly in the region between the two walls with a bulk velocity of $V$ in the $+x$-direction. The temperatures of the electrons and the ions are equal in the upstream. At the early stage of the simulation, particles that were located near the right wall were reflected by the wall and then interact with the incoming particles, i.e., the upstream plasma. This interaction causes some instability and eventually leads to the formation of a collisionless shock. Note that the frame of the simulation is the downstream rest frame; we observe the propagation of the shock from the right to the left in the downstream rest frame.

\section{B. Results}

Figure 3 shows the time evolution of the $x$-component of the electric field, $E_{x}$, averaged over the $y$-direction. We confirm that the electrostatic shock surely forms at around $\omega_{\text {pe }} t \sim 1000$ and propagates until $\omega_{\text {pe }} t \sim 2000$ at the constant velocity of $V_{\text {sh,d }}=1.2 \times 10^{-3} c$ measured in the downstream frame; the shock velocity measured in the upstream frame is given by $V_{\mathrm{sh}}=1.1 \times 10^{-2} \mathrm{c}$ and the shock Mach number is estimated as $M_{\mathrm{s}}=V_{\mathrm{sh}} / c_{\mathrm{s}}=3.2$.

After $\omega_{\text {pe }} t=2000$, however, the shock slows down and finally fades away. Figure 4 shows snapshots of the ion number density at $\omega_{\text {pe }} t=1500,3000$, and 5000. It is evident that oblique filamentary structures develop in front of the shock where the plasma is under the counter- 
streaming condition due to the existence of the reflected ions. We see that with the development of the filaments, the upstream plasma is strongly fluctuated and the shock structure is significantly modified. A similar structure was also observed in the simulation by Karimabadi, Omidi \& Quest [22]. Figure 5] shows the charge density at $\omega_{\text {pe }} t=3000$ and its power spectrum, which is directly related with the existence of the electrostatic modes. Comparing the region where the power is large in the power spectrum with that in the growth rate map obtained by the linear theory shown in Fig. 1, it is clear that these are generated by the electrostatic ion-ion instability. Note that, as is shown later, in fact the number density of the reflected ions is smaller than that of the incoming ions. The ratio is typically 0.6 [see Fig. 8 (d)]. However, the growth rate as a function of $k_{x}$ and $k_{y}$ (not shown here) is not significantly different from that of the symmetric beam case shown in Fig. 1] the maximum growth rate is obtained as $\Gamma=2.4 \times 10^{-3} \omega_{\text {pe }}$ at $|k|=7.6 \omega_{\mathrm{pe}} c^{-1}$ and $\theta=79^{\circ}$, which is only slightly smaller than the symmetric case.

Figure 6] shows the phase-space plots of the ions and electrons at $\omega_{\mathrm{pe}} t=1500,3000$, and 5000. The thermal velocity of the ions in the upstream region increases with time in both $x$ and $y$ directions, while the increase in the velocity dispersion of the electrons is relatively small. Figure 7 shows the time evolution of the velocity distributions of the ions within $143<x / \lambda_{\mathrm{e}}<145$, just in front of the shock front. The ions are heated especially in the $y$ direction because the electric field generated by the electrostatic ion-ion instability is almost directed in the $y$-direction. Thus, the anisotropy in the ion temperature increases with time.

There are two possible causes for the shock decay: one

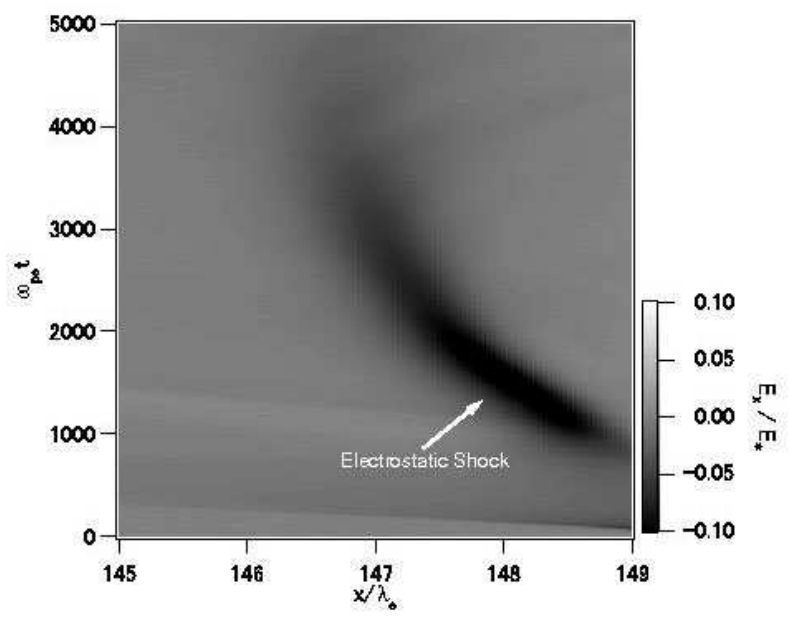

FIG. 3: Time evolution of the $x$-component of the electric field, $E_{x}$, averaged over the $y$-direction (gray-scale). The horizontal and vertical axes are the $x$-axis and the time, respectively. The electrostatic shock forms and propagates during $1000<\omega_{\text {pe }} t<2000$. However, after that time, the shock slows down and finally fades away. is the anisotropy in the ion temperature (or the decrease in the electron-to-ion temperature ratio), and the other is the fluctuation of the upstream plasma; both are caused by the electrostatic ion-ion instability. The time evolution of the temperatures immediately in front of the shock are shown in Fig. 8 together with that of the ratio of the reflected ion density to the incoming ion density. Here, the temperatures are calculated for the particles within the region in front of the shock given by

$$
x_{\mathrm{sh}}(t)-3 \lambda_{\mathrm{e}}<x<x_{\mathrm{sh}}(t)-1 \lambda_{\mathrm{e}},
$$

where we take $x_{\mathrm{sh}}(t)=-V_{\mathrm{sh}, \mathrm{d}}\left(t-1000 \omega_{\mathrm{pe}}^{-1}\right)+148.5 \lambda_{\mathrm{e}}$ and $V_{\mathrm{sh}, \mathrm{d}}=1.2 \times 10^{-3} \mathrm{c}$ from Fig. 3. For the ions, we distinguish the "incoming ions" that have $v_{x}>0$ and the "reflected ions" that have $v_{x}<0$, while the electrons are regarded as a single population because the mean (bulk) velocity of the electrons are negligible compared to their thermal velocity. Since the electrostatic shock is formed

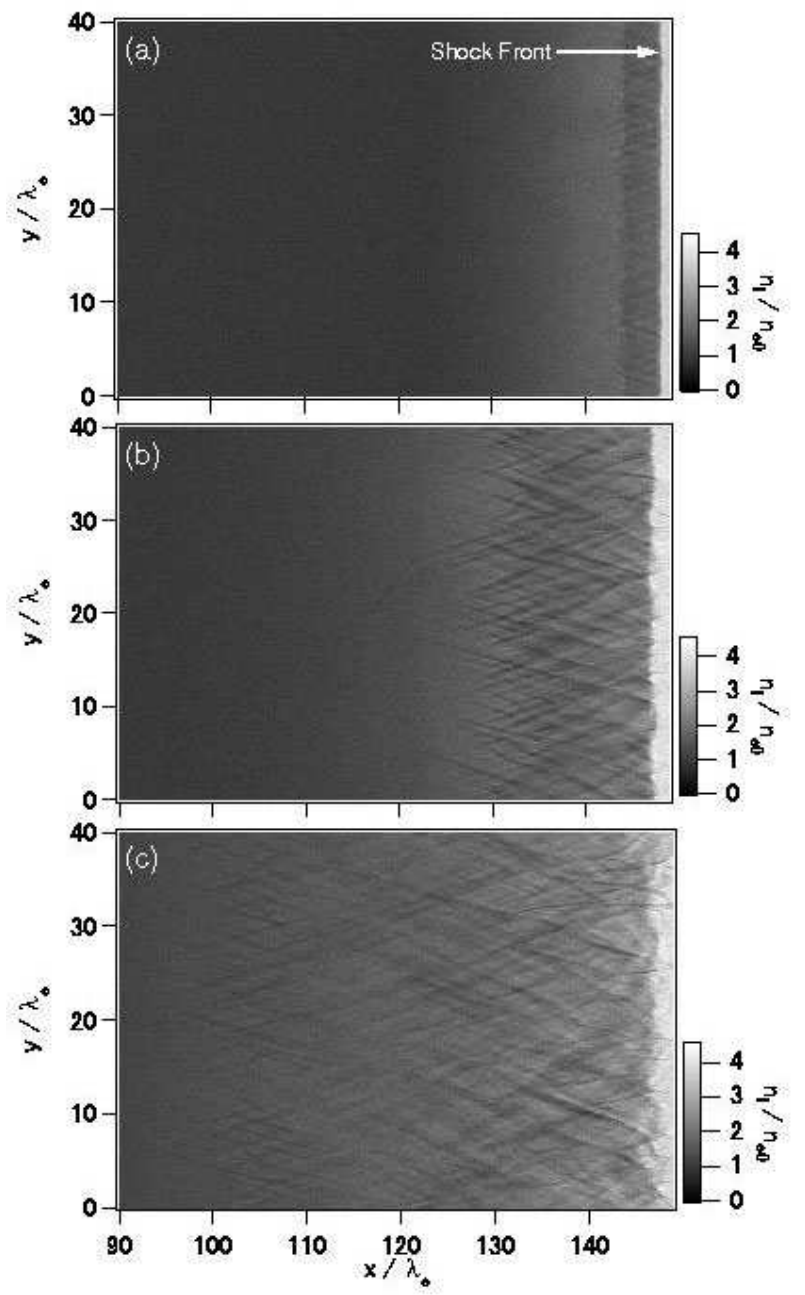

FIG. 4: Number density of the ions at $\omega_{\mathrm{pe}} t=$ (a) 1500, (b) 3000 , and (c) 5000. The horizontal and vertical axes are the $x$-axis and the $y$-axis in the electron skin depth, respectively. The electrostatic ion-ion instability, which generates the filamentary structure, develops with time. 
at $\omega_{\text {pe }} t \sim 1000$, the temperatures before that time are meaningless as those in front of the shock. We see that the electrons are heated in the $x$ and the $y$ directions in front of the shock immediately after the shock formation and then each component of the electron temperature keeps almost constant. On the other hand, the temperatures in the $y$-direction for both the incoming ions and

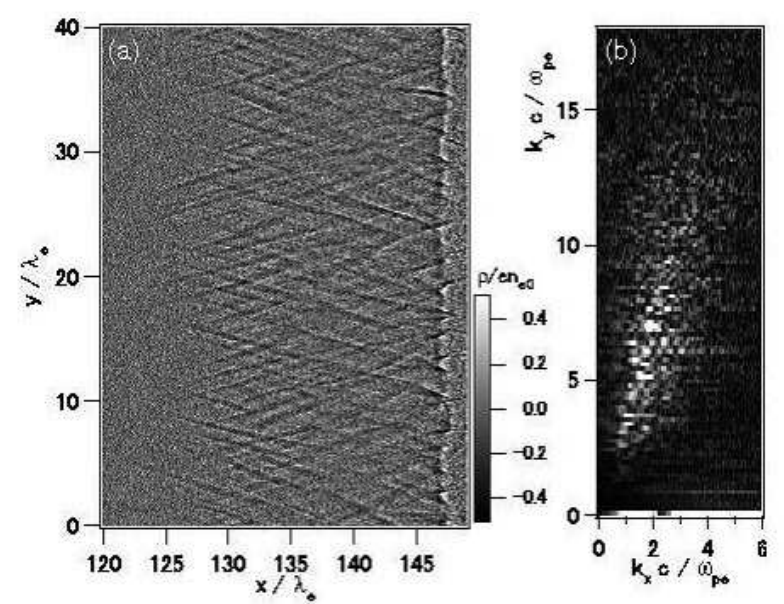

FIG. 5: (a) The charge density at $\omega_{\mathrm{pe}} t=3000$ and (b) its power spectrum. The horizontal and vertical axes in (b) are $k_{x}$ and $k_{y}$ in $\omega_{\mathrm{pe}} c^{-1}$, respectively. The portion where the electrostatic mode develops shown in the panel (b) is well agree with those obtained from the linear analysis shown in Fig. 1 .
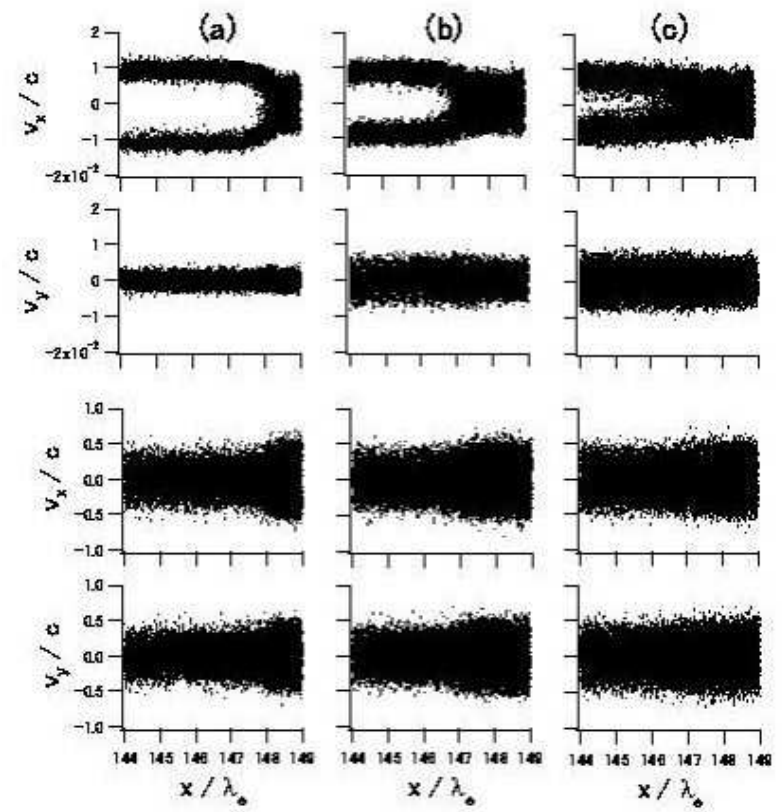

FIG. 6: Phase-space plots of the ions and electrons at $\omega_{\mathrm{pe}} t=$ (a) 1500 , (b) 3000, and (c) 5000: from top to bottom, the $x$ - $v_{x}$ and $x-v_{y}$ distributions of the ions and those of the electrons are shown. In the upstream of the shock, the ion heating is evident while the electron heating is small. the reflected ions rapidly increase with time within the period $1800<\omega_{\text {pe }} t<2300$, which coincides with the period of the shock decay, while the other components do not change significantly. The ion heating in the $y$ direction would be caused by the electrostatic ion-ion instability and results in the anisotropic temperatures in the ions as well as the reduction of the electron-to-ion temperature ratio.

To clarify whether the anisotropic temperature in the ions is the cause of the shock decay, we carried out another (quasi) one-dimensional simulation with an anisotropic ion temperature. The simulation code used is

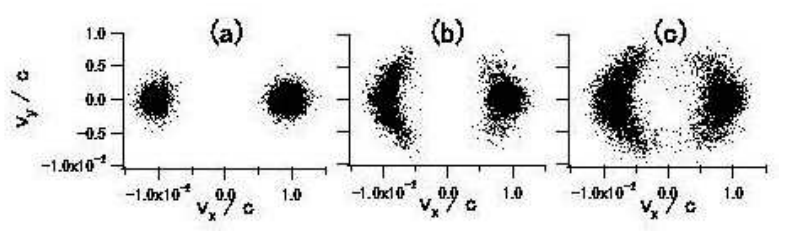

FIG. 7: Velocity distributions of the ions in the $v_{x}-v_{y}$ space within $143<x / \lambda_{\mathrm{e}}<145$ at $\omega_{\mathrm{pe}} t=$ (a) 1500, (b) 3000, and (c) 5000. The heating in the $y$-direction is remarkable.

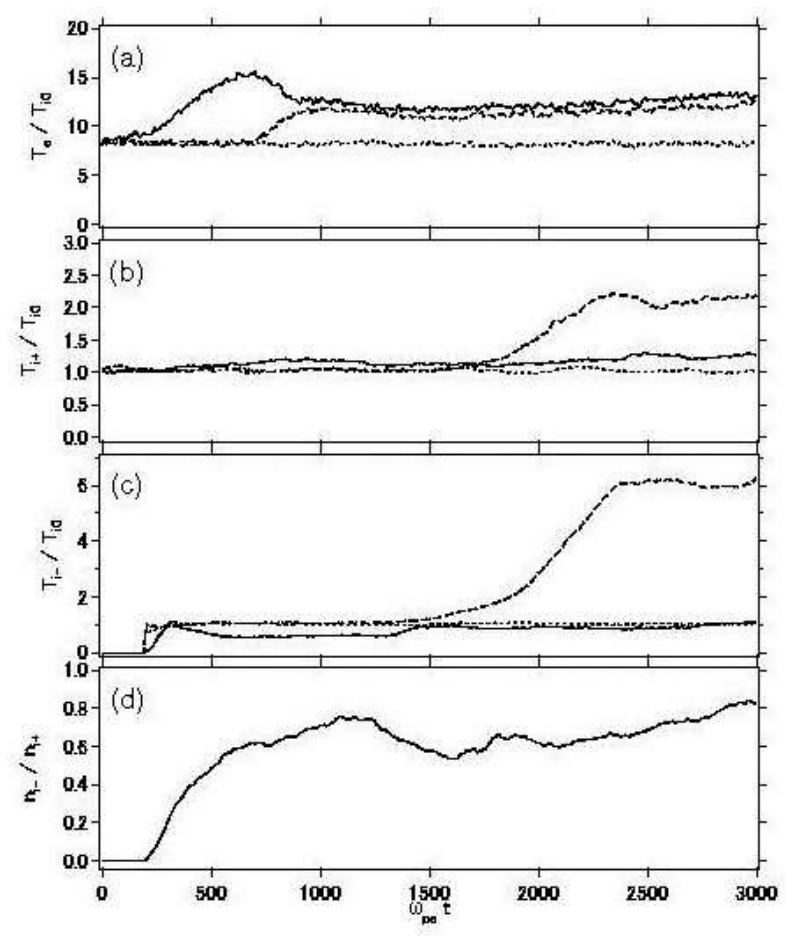

FIG. 8: Time development of the temperature in each direction immediately in front of the electrostatic shock normalized to the initial ion temperature $T_{i 0}$ : (a) the electrons, (b) the incoming ions, and (c) the reflected ions. The solid curves, the dashed curves, and the dotted curves show the $x, y$, and $z$ components, respectively. It is remarkable that the $y$ components of the temperatures of both ion populations rapidly increase within the period $1800<\omega_{\text {pe }} t<2300$. The panel (d) shows the time development of the number density ratio of the reflected ions to the incoming ions, $n_{i-} / n_{i+}$. 
the same two-dimensional code but we take only 8 grids in the $y$-direction so that the simulation is essentially onedimensional; in such a simulation, since the electrostatic ion-ion instability does not develop, we can investigate the pure effect of the anisotropy in the ion temperature on the shock formation. For the shock formation, the condition of the incoming ions is more important than that of the reflected ions and Fig. 8 (b) shows the typical anisotropic temperature of the incoming ions is given by $T_{\mathrm{i}, y} \sim 2 T_{\mathrm{i}, x}$ around the time of the shock decay. Here, as a more severe case, we take $T_{\mathrm{i}, y}=4 T_{\mathrm{i}, x}$ keeping the other parameters unchanged as in the two-dimensional simulation, that is, $T_{\mathrm{e}} / T_{\mathrm{i}, x}=9$. The time evolution of the electric field $E_{x}$ is shown in Fig.9(a). We found that the electrostatic shock forms under even this condition, where the shock speed measured in the upstream frame is $V_{\text {sh }} \sim 1.2 \times 10^{-2} c$ and the Mach number is given by $M_{\mathrm{s}} \sim 3.5$.

To confirm that the decrease in the electron-to-ion temperature ratio with isotropic ion temperature is also not the cause of the shock decay, we performed another quasi one-dimensional simulation with an isotropic ion temperature but the lower electron-to-ion temperature ratio of $T_{\mathrm{e}} / T_{\mathrm{i}}=4$. The result is shown in Fig. 9 (b) showing that the shock is also formed with even this temperature ratio at almost the same shock velocity and the Mach number as the anisotropic case. Thus, the anisotropic temperature in the ions nor the decrease in the temperature ratio are not the cause of the shock decay observed in the two-dimensional simulation.
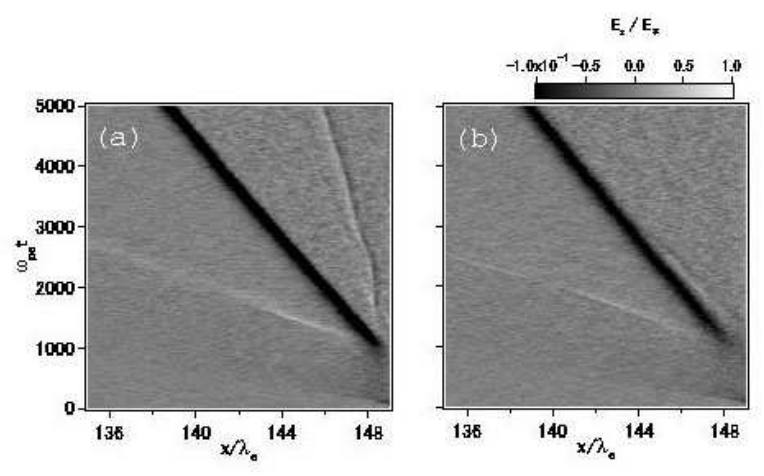

FIG. 9: Time evolutions of the $x$-component of the electric field, $E_{x}$, as in Fig. 3 obtained from quasi one-dimensional simulations (see text): (a) for the anisotropic ion temperature $T_{\mathrm{i}, y} / T_{\mathrm{i}, x}=4$ and $T_{\mathrm{e}} / T_{\mathrm{i}, x}=9$ and (b) for the isotropic ion temperature with $T_{\mathrm{e}} / T_{\mathrm{i}}=4$. The electrostatic shocks form and propagate without decaying in both cases.

For completeness, we also performed another quasi one-dimensional simulation with the same parameters as the two-dimensional one, that is, $T_{\mathrm{e}} / T_{\mathrm{i}}=9$, except the grid number in the $y$-direction. We found that the electrostatic shock forms and propagates without decaying in this case as well (not shown) confirming that the shock decay is a multi-dimensional effect.

Figure 10 shows the electrostatic potentials, the num- ber density of the incoming ions, and that of the reflected ions at $\omega_{\mathrm{pe}} t=2000$ for the two-dimensional simulation. The potentials are normalized by the upstream ion kinetic energy $\left(m_{\mathrm{i}} V^{2} / 2\right)$. It is evident that the large potential fluctuation, which reaches even a half of the shock potential, exists in front of the shock due to the electrostatic ion-ion instability. Because of this electrostatic potential, the densities of both incoming and reflected ions are significantly disturbed where the fluctuation pattern almost traces the waves of the electrostatic ion-ion instability. In particular, it can be seen that the portions where the incoming ions enter the "shock front" are different from those where the ions are reflected toward upstream from the "front". Figure 11] shows the $y$ - $v_{y}$ phase
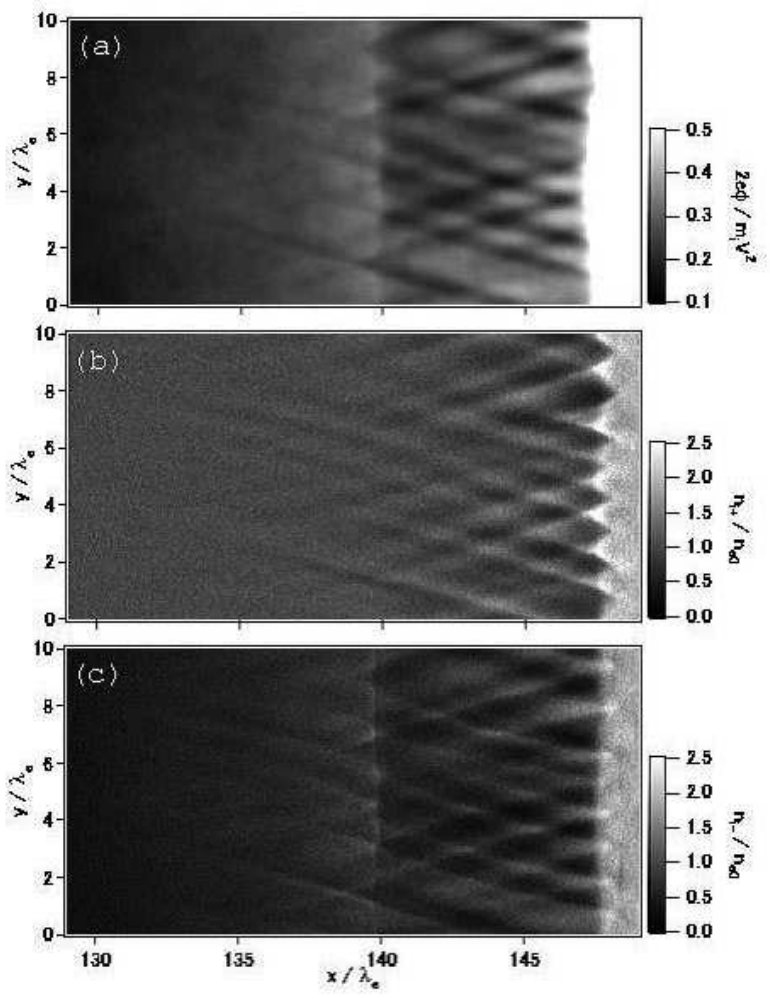

FIG. 10: Close-ups of (a) The electrostatic potentials, (b) the number density of the incoming ions $\left(n_{\mathrm{i}+}\right)$, and (c) that of the reflected ions $\left(n_{\mathrm{i}-}\right)$ at $\omega_{\mathrm{pe}} t=2000$ for the two-dimensional simulation. In (a), the electrostatic potential in front of the shock, which is generated by the electrostatic ion-ion instability, reaches even about a half of the shock potential.

space distribution of the ions around $x \sim 145.5 \lambda_{\mathrm{e}}$. The feature of the ion trapping [22, 31] is clear and therefore the fluctuation of the ion densities is a result of the nonlinear evolution of the electrostatic ion-ion instability. Thus, it can be concluded that the cause of the decay of the electrostatic shock is the large fluctuation in the ion density due to the electrostatic ion-ion instability.

In Fig.10 (c), we can also find another interesting feature in the reflected ion density that is seen as a discontinuity around $x \sim 140 \lambda_{\mathrm{e}}$, which could also be found in Fig. 4 (a). The $x-v_{x}$ phase space distribution of the 
ions is plotted in Fig. 12, As is shown in the panel (a), there is a structure in the phase space of the reflected ions around $x \sim 140 \lambda_{\mathrm{e}}$. The panel (b) shows the closeup of this structure. We see that some of the reflected ions are reflected again there in the opposite direction. This structure would also be another electrostatic shock in which the upstream region is the larger $x$ side. This 'secondary' electrostatic shock would be formed as a result of that the faster reflected ions run into the slower ones from behind.

As was shown in Sec. II, the beam-Weibel instability can develop in counter-streaming plasmas with the wave vector in the $y$-direction as well as the electrostatic ion-ion instability. However, the large anisotropy in the ion temperature $\left(T_{\mathrm{i}, y} / T_{\mathrm{i}, x} \sim 2\right.$ for the incoming ions and $T_{\mathrm{i}, y} / T_{\mathrm{i}, x} \sim 6$ for the reflected ions) caused by the electrostatic instability can affect the growth of the beam-Weibel instability. In addition, this temperature anisotropy itself may also cause the (ordinary) Weibel instability that has the wave vector in the $x$-direction. Here, let us consider the linear dispersion relation of these two Weibel-type instabilities in the anisotropic Maxwellian beam system given by the following zerothorder distribution functions:

$$
\begin{aligned}
& f_{0}^{(\mathrm{s})}\left(v_{x}, v_{y}, v_{z}\right)= \\
& \frac{n_{\mathrm{s}}}{\pi^{3 / 2} a_{\mathrm{s} x} a_{\mathrm{s} y} a_{\mathrm{s} z}} \exp \left[-\frac{\left(v_{x}-V_{\mathrm{s}}\right)^{2}}{a_{\mathrm{s} x}^{2}}-\frac{v_{y}^{2}}{a_{\mathrm{s} y}^{2}}-\frac{v_{z}^{2}}{a_{\mathrm{s} z}^{2}}\right]
\end{aligned}
$$

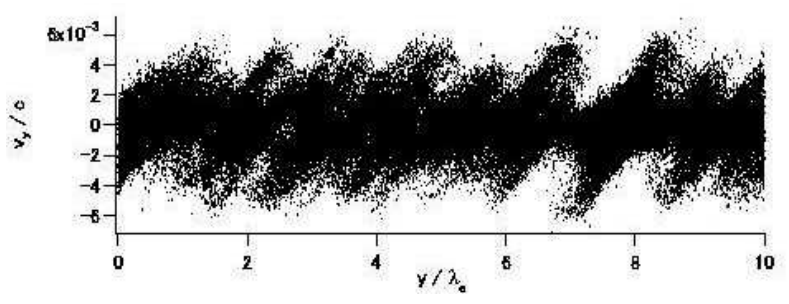

FIG. 11: The $y-v_{y}$ phase space distribution of the ions within $145.5<x / \lambda_{\mathrm{e}}<145.7$ at $\omega_{\mathrm{pe}} t=2000$ (c.f. Fig. 10). The feature of the ion trapping due to the nonlinear evolution of the electrostatic ion-ion instability is clear. This trapping results in the strong fluctuation in the incoming ions as is shown in Fig. 10(b) to destroy the shock structure.
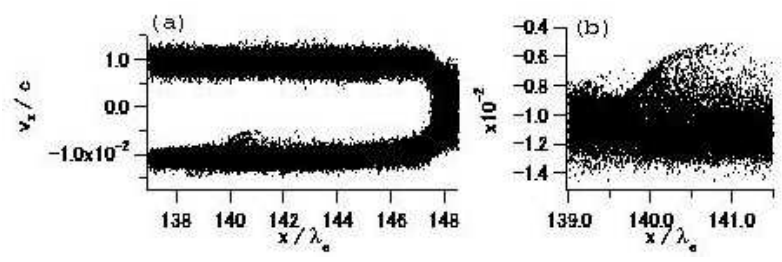

FIG. 12: (a) The $x-v_{x}$ phase space distribution of the ions within $0<y / \lambda_{\mathrm{e}}<0.4$ at $\omega_{\mathrm{pe}} t=2000$. (b) The close-up of the distribution of the reflected ions around $x \sim 140 \lambda_{\mathrm{e}}$. The "secondary" electrostatic shock exists in the reflected ion beam around $x=140 \lambda_{\mathrm{e}}$. where $a_{\mathrm{s} x}, a_{\mathrm{s} y}$ and $a_{\mathrm{s} z}$ are the anisotropic thermal velocities for the respective directions of the species s. In the following, we consider the beams are symmetrical for simplicity, that is, $n_{e+}=n_{e-}=n_{i+}=n_{i-}=n_{\mathrm{e} 0}$, $V_{\mathrm{e}+}=V_{\mathrm{i}+}=V$ and $V_{\mathrm{e}-}=V_{\mathrm{i}-}=-V$, as in Sec. II. Under this condition, the linear dispersion relation of the beam-Weibel instability with the wave vector in the $y$ direction is given by

$\omega^{2}-(k c)^{2}+\sum_{\mathrm{s}} \omega_{\mathrm{ps}}^{2}\left[\frac{a_{\mathrm{s} x}^{2}+2 V^{2}}{a_{\mathrm{s} y}^{2}}\left(1+\zeta_{\mathrm{s}} Z\left(\zeta_{\mathrm{s}}\right)\right)-1\right]=0$,

where $\zeta_{\mathrm{s}} \equiv \omega / k a_{\mathrm{s} y}$. On the other hand, the linear dispersion relation of the (ordinary) Weibel instability with the wave vector in the $x$-direction is given by

$$
\omega^{2}-(k c)^{2}+\sum_{\mathrm{s}} \omega_{\mathrm{ps}}^{2}\left[\frac{a_{\mathrm{s} y}^{2}}{a_{\mathrm{s} x}^{2}}\left(1+\eta_{\mathrm{s}} Z\left(\eta_{\mathrm{s}}\right)\right)-1\right]=0,
$$

where $\eta_{\mathrm{s}} \equiv\left(\omega / k-V_{\mathrm{s}}\right) / a_{\mathrm{s} x}$. (Note that when $V=0$, this relation is reduced to that derived by Weibel 24 without the background magnetic field.) Figure 13 shows the growth rates of the two modes. We take here $T_{\mathrm{i}, y} / T_{\mathrm{i}, x}=4, T_{\mathrm{i}, z}=T_{\mathrm{i}, x}$ and $T_{\mathrm{e}} / T_{\mathrm{i}, x}=9$ as typical values and the other parameters are the same as those of the initial condition of the two-dimensional simulation. As a reference, the growth rate for the isotropic beam case with $T_{\mathrm{e}} / T_{\mathrm{i}}=9$ discussed in Sec. [I] is also shown in the figure. We see that the growth rate of the beam-Weibel instability is reduced compared with that of the isotropic beam case due to the temperature anisotropy in the ions. Under this condition, the Weibel mode with the wave vector in the $x$-direction is stable and does not grow. (Of course, in the limit $V \rightarrow 0$, this mode should be unstable. We confirmed that it is unstable for $V<1.15 \times 10^{-3} c$ for the same parameters.)

Figure 14]shows the $z$-component of the magnetic field, $B_{z}$, obtained from the two-dimensional simulation at $\omega_{\mathrm{pe}} t=1500,3000$, and 5000. We see that the magnetic field grows with time with a wavelength comparable with that derived by the linear analysis $\left(\sim 40 \lambda_{\mathrm{e}}\right)$, although it may be affected by the periodic boundary condition because the wavelength is comparable with the system length in the $y$-direction. At this time $\left(\omega_{\mathrm{pe}} t \sim 5000\right)$, the typical magnetic field strength is $|B| / B_{*} \sim 2 \times 10^{-2}$ and the corresponding ion gyro-radius is given by $\sim 9 \times 10^{2} \lambda_{\mathrm{e}}$; therefore, the ions are hardly deflected by this magnetic field. However, in the Weibel-type instability, the magnetic field is generated by the current filaments and the current filaments coalesce each other to grow larger filaments with stronger magnetic fields until saturation [see e.g., 32]. Although, because of the limitation of the simulation box, we cannot study the further evolution of the filaments in this simulation, in the real world or in much larger simulations, the magnetic field would grow much stronger with the coalescence of the filaments and, long after the electrostatic shock vanished, it would become strong enough to form the "Weibel-mediated" shocks, a 
kind of collisionless shocks that dissipates the upstream particle kinetic energy via the magnetic field generated by the instability in the shock transition region.

As is reported in our previous paper of Ref. [29], we have performed a PIC simulation of the Weibel mediated shock with the larger flow velocity $(V=0.45 c)$ and the smaller mass ratio $\left(m_{\mathrm{i}} / m_{\mathrm{e}}=20\right)$ with the equal temperatures for the electrons and the ions. (In that simulation, the electrostatic shock and the electrostatic ion-ion instability do not develop.) To illustrate the formation of the Weibel-mediated shock qualitatively, the time evolutions of the ion number density and the magnetic field ( $z$-component) are shown in Fig.15. The figures show the ion current filaments develop with time due to the beamWeibel instability and finally the Weibel-mediated shock forms at $\omega_{\mathrm{pe}} t=2000$. Note that due to the mass ratio and the flow velocity, the temporal and spatial scales are different from those in the system with the real mass ratio of $m_{\mathrm{i}} / m_{\mathrm{e}}=1836$ and the slower flow speed $\sim 3000$ $\mathrm{km} \mathrm{s}^{-1}$. Returning to the situation of the present study, the ion current filaments developing around the electrostatic shock (Fig. 114) would also grow further and finally will evolve into the Weibel-mediated shock in the real world or in the simulations with much larger spatial and temporal scales.

\section{CONCLUSION}

We have carried out a two-dimensional electromagnetic PIC simulation for the case of counter-streaming plasmas at a relatively high flow velocity with a large electron-to-

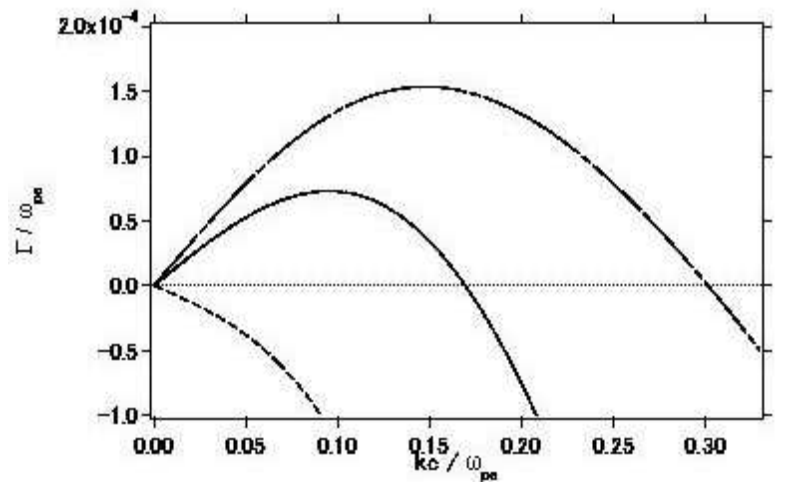

FIG. 13: The linear growth rates of the two Weibeltype instabilities in the anisotropic Maxwellian beam system $\left(T_{\mathrm{i}, y} / T_{\mathrm{i}, x}=4\right.$ and $\left.T_{\mathrm{e}} / T_{\mathrm{i}, x}=9\right)$ : the beam-Weibel instability with the wave vector in the $y$-direction (solid curve) and the (ordinary) Weibel instability with the wave vector in the $x$-direction (dashed curve). The dot-dashed curve shows the growth rate of the beam-Weibel instability in the isotropic Maxwellian beam system as in Sec. II (i.e., $T_{\mathrm{i}, y} / T_{\mathrm{i}, x}=1$ and $\left.T_{\mathrm{e}} / T_{\mathrm{i}}=9\right)$ as a reference. The growth rate of the beam-Weibel instability is reduced due to the temperature anisotropy in the ions. The Weibel instability in the $x$ direction is stable in this case. ion temperature ratio of 9 . At first we have confirmed that the electrostatic shock forms in the early time evolution as was shown in the previous works 3 , 14]. We also confirmed that the electrostatic ion-ion instability develops in front of the shock due to the counter-streams of the ions [22]. Then, we found that the electric field generated by this instability results in the strong fluctuation in the ion density and finally leads to destroy the shock itself. It was also found that the electromagnetic beam-Weibel instability develops much slower than the electrostatic instability but it becomes predominant in the later time. This suggests the possibility that the Weibel-mediated collisionless shock is formed according to the scenario shown in Kato \& Takabe [29] long after the electrostatic shock disappears. It was also observed that the secondary electrostatic shock forms in the reflected ions in front of the primary electrostatic shock.

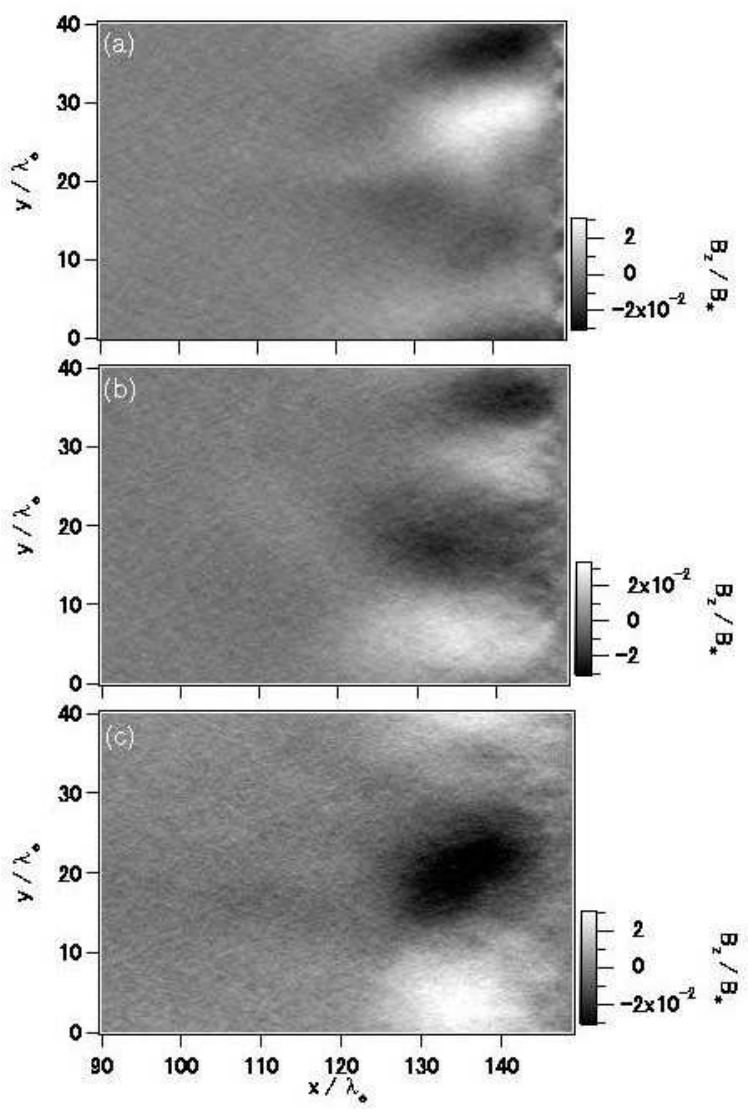

FIG. 14: The $z$-component of the normalized magnetic field, $B_{z} / B_{*}$, at $\omega_{\mathrm{pe}} t=$ (a) 1500 , (b) 3000, and (c) 5000 for the two-dimensional simulation, where $B_{*} \equiv c\left(4 \pi n_{\mathrm{e} 0} m_{\mathrm{e}}\right)^{1 / 2}$. The magnetic field is generated by the beam-Weibel instability in front of the shock and it grows with time during the simulation. 


\section{Acknowledgments}

We thank Y. Ohira for helpful discussions. We also thank Y. Sakawa, Y. Kuramitsu and T. Morita for the discussion of their experimental data. This work was supported in part by the Ministry of Education, Culture, Sports, Science and Technology (MEXT), Grant-in-Aid for Young Scientists (B) (T.N.K.: 20740136). Numerical computations were carried out at Cybermedia Center, Osaka University.

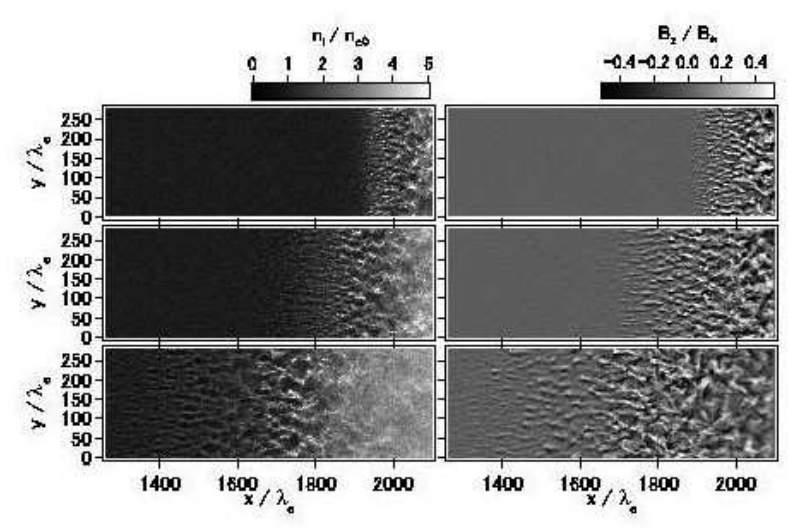

FIG. 15: PIC simulation of the Weibel-mediated shock for showing the formation of the shock qualitatively with parameters of $m_{\mathrm{i}} / m_{\mathrm{e}}=20$ and $V=0.45 c$. The left panel shows the ion number density normalized by the upstream value, $n_{i} / n_{\mathrm{e} 0}$, and the right panel shows the $z$-component of the normalized magnetic field, $B_{z} / B_{*}$, from top to bottom at $\omega_{\mathrm{pe}} t=500$, 1000 , and 2000, respectively. Due to the magnetic field generated by the beam-Weibel instability, a kind of collisionless shock, which is called the Weibel-mediated shock, is formed at $\omega_{\text {pe }} t \sim 2000$.

[1] S. S. Moiseev and R. Z. Sagdeev, J. Nucl. Energy Part C 5, 43 (1963).

[2] F. F. Chen, Introduction to Plasma Physics (Plenum Press, New York, 1974).

[3] R. J. Mason, Phys. Fluids 15, 845 (1972).

[4] R. J. Taylor, D. R. Baker, and H. Ikezi, Phys. Rev. Lett. 24, 206 (1970).

[5] H. Ikezi, T. Kamimura, M. Kako and K. E. Lonngren, Phys. Fluids 16, 2167 (1973).

[6] H. Bailung, Y. Nakamura, and Y. Saitou, Phys. Plasmas 15, 052311 (2008).

[7] T. Takeuchi, S. Iizuka, and N. Sato, Phys. Rev. Lett. 80, 77 (1998).

[8] D. B. Cohn, and K. R. MacKenzie, Phys. Rev. Lett. 28, 656 (1972).

[9] D. W. Koopman and D. A. Tidman, Phys. Rev. Lett. 18, 533 (1967).

[10] L. Romagnani, S. V. Bulanov, M. Borghesi, P. Audebert, J. C. Gauthier, K. Löwenbrück, A. J. Mackinnon, P. Patel, G. Pretzler, T. Toncian, and O. Willi, Phys. Rev. Lett. 101, 025004 (2008).
[11] T. Morita, Y. Sakawa, Y.Kuramitsu, S. Dono, H. Aoki, H. Tanji, T. N. Kato, Y. T. Li, Y. Zhang, X. Liu et al., "Collisionless shock generation in high-speed counterstreaming plasma flows by a high-power laser" submitted to Phys. Rev. Lett.

[12] F. S. Mozer, Geophys. Res. Lett. 8, 823 (1981).

[13] R. J. Mason, Phys. Fluids 14, 1943 (1971).

[14] D. W. Forslund and C.R. Shonk, Phys. Rev. Lett. 25, 1699 (1970).

[15] G. Sorasio, M. Marti, R. Fonseca and L. O. Silva, Phys. Rev. Lett. 96, 045005 (2006).

[16] T. E. Stringer, J. Nucl. Energy Part C 6, 267 (1964).

[17] T. Ohnuma and Y. Hatta, Kakuyugo-Kenkyu 15, 637 (1965).

[18] B. D. Fried and A. Y. Wong, Phys. Fluids 9, 1084 (1966).

[19] S. P. Gary and N. Omidi, J. Plasma Phys. 37, 45 (1987).

[20] D. W. Forslund and C. R. Shonk, Phys. Rev. Lett. 25, 281 (1970).

[21] D. Grésillon, F. Doveil, and J. M. Buzzi, Phys. Rev. Lett. 34, 197 (1975).

[22] H. Karimabadi, N. Omidi, and K. B. Quest, Geophys. 
Res. Lett. 18, 1813 (1991).

[23] Y. Ohira and F. Takahara, Astrophys. J. 688, 320 (2008).

[24] E. S. Weibel, Phys. Rev. Lett. 2, 83 (1959).

[25] B. D. Fried, Phys. Fluids 2, 337 (1959).

[26] B. D. Fried and S. D. Conte, The plasma dispersion function (Academic Press, New York, 1961).

[27] C. K. Birdsall and A. B. Langdon, Plasma Physics via Computer Simulation (IOP Publishing, Bristol, 1991).

[28] T. N. Kato, Astrophys. J. 668, 974 (2007).

[29] T. N. Kato and H. Takabe, Astrophys. J. Lett. 681, L93
(2008)

[30] H. Takabe, T. N. Kato, Y. Sakawa, Y. Kuramitsu, T. Morita, T. Kadono, K. Shigemori, K. Otani, H. Nagatomo, T. Norimatsu et al., Plasma Phys. Control. Fusion 50, 124057 (2008).

[31] N. Omidi and K. Akimoto, J. Geophys. Res. 93, 14,725 (1988).

[32] T. N. Kato, Phys. Plasmas 12, 080705 (2005). 